\title{
Diffusion of Behavior and Equilibrium Properties in Network Games
}

\author{
By Matthew O. Jackson And LeEat Yariv*
}

Situations in which agents' choices depend on choices of those in close proximity, be it social or geographic, are ubiquitous. Selecting a new computer platform, signing a political petition, or even catching the flu are examples in which social interactions have a significant role. While some behaviors or states propagate and explode within the population (e.g., Windows OS, the HIV virus) others do not (e.g., certain computer viruses). ${ }^{1}$ Our goal in this paper is twofold. First, we provide a general dynamic model in which agents' choices depend on the underlying social network of connections. Second, we show the usefulness of the model in determining when a given behavior expands within a population or disappears as a function of the environment's fundamentals.

We study a framework in which agents face a choice between two actions, 0 and 1 (e.g., whether to pursue a certain level of education, switch to Linux OS, etc.). Agents are linked through a social network, and an agent's payoffs from each action depend on the number of neighbors she has and her neighbors' choices. The diffusion process is defined so that at each period, each agent best responds to the actions taken by her neighbors in the previous period, assuming that her neighbors follow the population distribution of actions (a mean-field approximation). Steady states correspond to equilibria of the static game. Under some simple conditions, equilibria take one of two forms. Some are stable, so that a slight perturbation to any such equilibrium

\footnotetext{
* Jackson: Department of Economics, Stanford University, Stanford CA 94305 (e-mail: jacksonm@stanford.edu); Yariv: Division of the Humanities and Social Sciences, Caltech, Pasadena, CA 91125 (e-mail: lyariv@hss.caltech. edu). We are grateful for financial support from the Center for Advanced Studies in the Behavioral Sciences and the Guggenheim Foundation. We thank Tanya Rosenblat for a helpful discussion of the paper.

${ }^{1}$ See Everett M. Rogers (1995), as well as virus prevalence data at http://www.virusbtn.com/ and summary statistics in Romualdo Pastor-Satorras and Alessandro Vespignani (2000).
}

would lead the diffusion process to converge back to that equilibrium point. Other equilibria are unstable, so that a slight change in the distribution of actions leads to a new distribution of actions and eventually to a stable steady state. We call such equilibria tipping points. We analyze how the environment's fundamentals (cost distribution, payoffs, and network structure) affect the set of equilibria, and characterize the adoption patterns within the network.

The paper relates to recent work on network games and network diffusion, including work by Stephen Morris (2000); Pastor-Satorras and Vespignani (2000); Mark E. J. Newman (2002); Dunia López-Pintado (2004); Jackson and Brian W. Rogers (2007); Jackson and Yariv (2005); and Andrea Galeotti et al. (2005, henceforth GGJVY). Its contribution is in characterizing diffusion of strategic behavior and analyzing the stability properties of equilibria, and employing methods that allow us to make comparisons across general network structures and settings. Given that social networks differ substantially and systematically in structure across settings (e.g., ethnic groups, professions, etc.), understanding the implications of social structure on diffusion is an important undertaking for a diverse set of applications.

\section{The Model}

\section{A. Social Networks and Payoffs}

We consider a set of agents and capture the social structure by its underlying network. We model the network through the distribution of the number of neighbors, or degree, that each agent has. Agent $i$ 's degree is denoted $d_{i}$. The fraction of agents in the population with $d$ neighbors is described by the degree distribution $P(d)$ for $d=0,1, \ldots, D$ (with the possibility that $D=\infty)$, where $\sum_{d=1}^{D} P(d)=1$.

Let $\widetilde{P}(d) \equiv P(d) d / \bar{d}$, where $\bar{d}=E_{P}[d]=$ $\sum_{d} P(d) d$. This is a standard calculation of the probability of the degree of an agent conditional 
on that agent being at the end of a randomly chosen link in the network.

Agents each have a choice between taking an action 0 or an action 1 . Without loss of generality, we consider the action 0 to be the default behavior (for example, the status-quo technology). Agent $i$ has a cost of choosing 1, denoted $c_{i}$. Costs are randomly and independently distributed across society according to a distribution $H^{c}$ that we assume to be atomless. We normalize the payoff from taking the action 0 to be $0 .^{2}$ Agent $i$ 's payoff from adopting behavior 1 when $i$ has $d_{i}$ neighbors and expects them each independently to choose 1 with a probability $x$ is $v\left(d_{i}, x\right)-c_{i}$. Therefore, $i$ prefers action 1 if $c_{i} \leq v\left(d_{i}, x\right)$.

We illustrate the generality of the framework by noting a few special cases:

- $v(d, x)=u(d x)$ - an agent's payoffs are a function of the expected number of neighbors adopting the action 1 . This corresponds to the framework analyzed in GGJVY.

- $v(d, x)=u(x)$-agents care only about the average play of their neighbors. Network structure does not enter.

- $v(d, x)$ is a step function, for instance, taking one value if $x$ lies below a threshold (possibly depending on $d$ ), and taking another value if $x$ exceeds the threshold.

\section{B. Bayesian Equilibrium}

We consider symmetric Bayesian equilibria of the network game:

- Each agent $i$ knows only her own degree $d_{i}$ and cost $c_{i}$, the distribution of degrees in the population, and assumes that degrees and cost parameters are independently allocated. Thus, the game is a Bayesian game in the Harsanyi sense where types are given by degrees and costs.

- The play is symmetric in that any agent perceives the distribution of play of each of her

\footnotetext{
${ }^{2}$ In Jackson and Yariv (2006), we analyze the more general case in which $H^{c}$ may contain atoms, and payoffs to the action 0 may depend on an agent's degree. The latter is important for welfare implications, but the normalization here is without loss of generality for strategic considerations.
}

neighbors to be independent and to correspond to the distribution of play in the population. ${ }^{3}$

Existence of symmetric Bayesian equilibria follows from standard arguments. In cases where $v$ is nondecreasing in $x$ for each $d$, existence is a direct consequence of Tarski's Fixed Point Theorem, and then there exists an equilibrium in pure strategies. In other cases, provided $v$ is continuous in $x$ for each $d$, we find a fixed point by appealing to standard fixed point theorems (e.g., Kakutani) and admitting mixed strategies.

A simple equation is sufficient to characterize equilibria. Let $x$ be the probability that a randomly chosen neighbor chooses the action 1 . Then $H(d, x) \equiv H^{c}[v(d, x)]$ is the probability that a random (best responding) neighbor of degree $d$ chooses the action 1 . It must be that

$$
x=\phi(x) \equiv \sum_{d} \widetilde{P}(d) H(d, x) .
$$

Equation 1 characterizes equilibria in the sense that any symmetric equilibrium results in an $x$ which satisfies the equation, and any $x$ that satisfies the equation corresponds to an equilibrium where type $\left(d_{i}, c_{i}\right)$ chooses 1 if and only if $c_{i} \leq v\left(d_{i}, x\right)$. Given that equilibria can be described by their corresponding $x$, we often refer to some value of $x$ as being an equilibrium.

\section{A Diffusion Process}

Consider a diffusion process governed by best responses in discrete time. At time $t=0$, a fraction $x^{0}$ of the population is exogenously and randomly assigned the action 1 , and the rest of the population is assigned the action 0 . At each time $t>0$, each agent, including the agents assigned to action 1 at the outset, best responds to the distribution of agents choosing the action 1 in period $t-1$, presuming that their neighbors will be a random draw from the population.

\footnotetext{
${ }^{3}$ This is an extension of the concept from GGJVY, where agents have identical costs. The equilibrium is symmetric in that it depends only on an agent's type $\left(d_{i}, c_{i}\right)$, and not her label $i$.
} 
Let $x_{d}^{t}$ denote the fraction of those agents with degree $d$ who have adopted behavior 1 at time $t$, and let $x^{t}$ denote the link-weighted fraction of agents who have adopted the behavior at time $t$. That is, $x^{t}=\sum_{d} \widetilde{P}(d) x_{d}^{t}$ and $x_{d}^{t}=H\left(d, x^{t-1}\right)$. Therefore, $x^{t}=\Sigma_{d} \widetilde{P}(d) H\left(d, x^{t-1}\right)$.

If payoffs exhibit complementarities, then convergence of behavior from any starting point is monotone, either upward or downward. Once an agent (voluntarily) switches behaviors, the agent will not want to switch back. Thus, although best responses are myopic, any changes in behavior are equivalently forward looking. Any rest point of the system corresponds to a static Bayesian equilibrium of the system. If actions are strategic substitutes, convergence may not be guaranteed for all starting points. Our results will still be useful in characterizing the potential rest points, or equilibria, of such systems, however.

\section{Equilibrium Structure}

\section{A. Multiplicity}

The multiplicity of equilibria is determined by the properties of $\phi$, which, in turn, correspond to properties of $\widetilde{P}$ and $H{ }^{4}$ In general, as long as the graph of $\phi(x)$ crosses the 45 degree line only once, there is a unique equilibrium (see Figure 1). There is a conceptual connection between our analysis and the recent literature on global games identifying forms of heterogeneity guaranteeing uniqueness when a game with complementarities admits multiple equilibria (see Morris and Hyun Shin 2003). The heterogeneity determining uniqueness in our setup is introduced through the cost agents experience, as well as through the different degrees agents have. Our analysis allows us to study the set of stable and unstable equilibria, regardless of multiplicity.

\section{B. Stability}

Some equilibria are robust to small perturbations and are therefore stable, while other equilibria are not robust and, from them, small perturbations lead to significant changes in the

\footnotetext{
${ }^{4}$ Jackson and Yariv (2006) discuss multiplicity and when 0 is an equilibrium point.
}

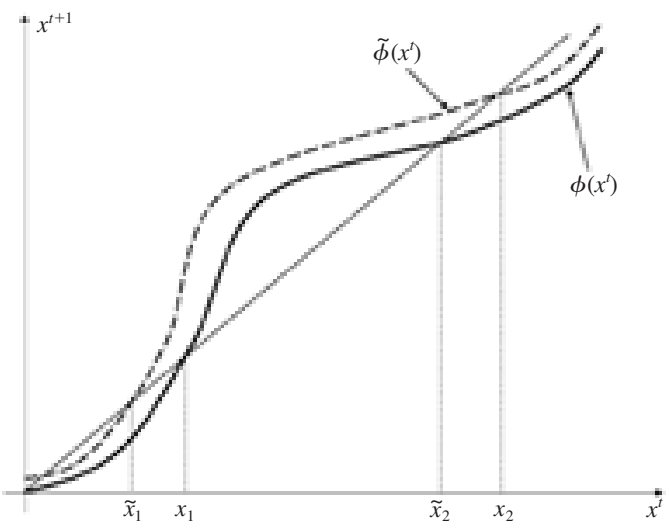

Figure 1. The Effects of Shifting $\phi(x)$ Pointwise

distribution of play in the population. These are captured in the following definitions.

DEFINITION 1 (Stability and Tipping): An equilibrium $x$ is stable if there exists $\varepsilon^{\prime}>0$ such that $\phi(x-\varepsilon)>x-\varepsilon$ and $\phi(x+\varepsilon)<x+\varepsilon$ for all $\varepsilon^{\prime}>\varepsilon>0$. An equilibrium $x$ is unstable or a tipping point if there exists $\varepsilon^{\prime}>0$ such that $\phi(x-\varepsilon)<x-\varepsilon$ and $\phi(x+\varepsilon)>x+\varepsilon$ for all $\varepsilon^{\prime}>\varepsilon>0$.

DEFINITION 2 (Regular Environment): $A n$ environment is regular if all fixed points are either stable or unstable, and H is continuous.

DEFINITION 3 (Greater Diffusion): One environment, with corresponding mapping $\widetilde{\phi}(x)$, generates greater diffusion than another with corresponding mapping $\phi(x)$, if for any stable equilibrium of the latter there exists a (weakly) higher stable equilibrium of the former, and for any unstable equilibrium of the latter there is either a (weakly) lower unstable equilibrium of the former or else $\widetilde{\phi}(0)>0$.

An environment has greater diffusion than another if its tipping points are lower, thus making it easier to get diffusion started, and its stable equilibria are higher, and so the eventual resting points are higher.

PROPOSITION 1: Consider $\bar{\phi}$ and $\phi$ corresponding through (1) to two regular environments. If $\bar{\phi}(x) \geq \phi(x)$ for each $x$, then $\bar{\phi}$ generates greater diffusion than $\phi$. 
Proposition 1 implies that a small upward shift in a (continuous) $\phi$ leads to locally lower tipping points and higher stable equilibria, as illustrated in Figure 1.

\section{Comparative Statics}

Given that $\phi(x)=\sum_{d} \widetilde{P}(d) H^{c}(v(d, x))$, and Proposition 1, we can deduce much about changes in the structure of equilibria by considering changes in fundamentals, costs, returns, and network structure, that shift $\phi(x)$ in a particular direction for all $x$. We refer the reader to Jackson and Yariv (2006) for omitted proofs.

\section{A. Changes in Cost Distribution}

We consider increases in costs in terms of first-order stochastic dominance (FOSD) shifts of $H^{c}$.

PROPOSITION 2 (Increasing Costs): If $\bar{H}^{c}$ FOSD $H^{c}$, then the corresponding $\bar{\phi}(x), \phi(x)$ satisfy $\bar{\phi}(x) \leq \phi(x)$ for each $x$. Thus, if $\bar{H}^{c}, H^{c}$, (given $v$ ) correspond to two regular environments, then $H^{c}$ generates greater diffusion than $\bar{H}^{c}$.

As costs increase, agents are generally less prone to take action 1, and so tipping points are shifting up and stable equilibria are shifting down.

Note that if $v(d, x)$ is nondecreasing in $x$, then if $x^{*}$ is a stable equilibrium under $H^{c}$ and $\bar{x}^{*} \leq x^{*}$ is a stable equilibrium under $\bar{H}^{c}$, expected utility of all agents goes up and expected welfare under $H^{c}$, when $x^{*}$ is played, is higher than under $\bar{H}^{c}$, when $\bar{x}^{*}$ is played.

\section{B. Changes in Network Structure}

We consider two types of changes to the network architecture. The first pertains to the number of expected neighbors each agent has. The second relates to the heterogeneity of connectedness within the population.

PROPOSITION 3 (FOSD Shifts): If $\widetilde{P}$ FOSD $\widetilde{P}^{\prime}$ and $H(d, x)$ is nondecreasing (nonincreasing) in $d$ for all $x$, then $\phi(x) \geq \phi^{\prime}(x)\left[\phi(x) \leq \phi^{\prime}(x)\right]$ for each $x$. Thus, if the environments corresponding to $P$ and $P^{\prime}$ are regular, then $P$ generates greater (lesser) diffusion than $P^{\prime}$.
To gain intuition, consider a case in which $v(d, x)$ is nondecreasing in $d$. Here, any symmetric equilibrium entails higher-degree agents choosing action 1 with higher probability. Start with any such equilibrium under $P^{\prime}$ and consider a shift to $P$ for which $\widetilde{P}$ FOSD $\widetilde{P}^{\prime}$. Without any change in strategies, each agent would perceive her neighbors to be more likely to have higher degrees. Thus, a best response would entail a greater propensity to choose the action 1. Iterating best responses converges to an equilibrium involving a (weakly) higher rate of agents of each type choosing the action 1 . It is easier to get the action 1 adopted and tipping points are lower. ${ }^{5}$

As for welfare, suppose $v(d, x)$ is nondecreasing in $x$. A FOSD change in the degree distribution generates higher expected payoffs corresponding to stable equilibria for agents of any given type. If the underlying degree distribution itself is shifted in the sense of FOSD (i.e., $P$ FOSD $P^{\prime}$ ), then more weight is shifted to higher expected payoff agents and overall welfare increases. This condition is naturally satisfied when, e.g., $P$ is a simple translation of the distribution $P^{\prime}$. Unfortunately, more general forms of FOSD shifts in the distribution of neighbors' degrees do not always correspond to FOSD shifts in the original degree distribution and so welfare implications are, in general, ambiguous. ${ }^{6}$

We now consider changes in the heterogeneity of connectedness through mean-preserving spreads of the degree distribution.

PROPOSITION 4 (MPS in P): If $H(d, x)$ is nondecreasing and convex (nonincreasing and concave) in $d$, then $P$ is a MPS of $P^{\prime}$ implies that $\phi(x) \geq \phi^{\prime}(x)\left[\phi(x) \leq \phi^{\prime}(x)\right]$ for all $x$, and so $P$ generates greater (lesser) diffusion than $P^{\prime}$. Furthermore, if $v(d, x)$ is convex (concave) in $d$ and nondecreasing in $x$, then if $x^{*}$ is a stable equilibrium under $P$ and $\bar{x}^{*} \leq x^{*}\left(\bar{x}^{*} \geq x^{*}\right)$

\footnotetext{
${ }^{5}$ A related result appears in GGJVY, but with several differences. That result does not distinguish between stable and unstable equilibria and applies only to a special class of payoff functions, but that result applies to more general action spaces (in the case where $H$ is nondecreasing).

${ }^{6}$ See GGJVY and Jackson and Yariv (2006) for discussion on this point and examples.
} 
is a stable equilibrium under $P^{\prime}$, the expected welfare under $P$ when $x^{*}$ is played is higher (lower) than under $P^{\prime}$ when $\bar{x}^{*}$ is played.

The greater diffusion result follows from the definition of MPS and $\phi(x)$. Regarding welfare, let $\bar{x}^{*}, x^{*}$ be given as stable points satisfying the Proposition's statement. The welfare level under $P$ when $x^{*}$ is played is $\sum_{d} v\left(d, x^{*}\right) P(d)$. Now, $\sum_{d} v\left(d, x^{*}\right) P(d) \geq \sum_{d} v\left(d, \bar{x}^{*}\right) P(d)$ since $v$ is nondecreasing, and this in turn is greater than or equal to $\sum_{d} v\left(d, \bar{x}^{*}\right) P^{\prime}(d)$, since $P$ is a MPS of $P^{\prime}$. The claim then follows.

Note that this result implies that if $H(d, x)$ is nondecreasing and convex, then power, Poisson, and regular degree distributions with identical means generate corresponding values of $\phi^{\text {power }}, \phi^{\text {Poisson }}$, and $\phi^{\text {regular }}$ such that $\phi^{\text {power }}(x) \geq$ $\phi^{\text {Poisson }}(x) \geq \phi^{\text {regular }}(x)$ for all $x$. This is consistent with the simulation-based observations regarding tipping points in the epidemiology literature (see Pastor-Satorras and Vespignani 2000).

Proposition 4 is useful in identifying the structure of optimal networks. Indeed, suppose we ask which $P$ with a given average $\bar{d}$ and support in $1, \ldots, \bar{D}$ maximizes $\phi(x)=\sum_{d} H(d, x)(P(d) d /$ $\bar{d})$. If we have a $P$ which maximizes this pointwise, then we know that it leads to greater diffusion than any other $P$. It follows directly that:

COROLLARY 5 (Optimal Networks): If $H(d, x)$ is nondecreasing and convex in $d$, then the $P$ that maximizes diffusion (under our greater diffusion partial ordering), is one which has weight only on degree 1 and $\bar{D}$ (in proportions to yield average degree $\bar{d})$. If $H(d, x)$ is nonincreasing and concave in $d$, then the $P$ that maximizes diffusion (under our greater diffusion partial ordering), is a regular network with full weight on degree $\bar{d}$.

\section{Changes in Returns to Adoption}

We now contemplate changes in the returns to the action 1 and their effects on the eventual adoption rate. This is interesting for a wide range of applications, e.g., directed advertising in marketing, optimal immunization processes in epidemiology, etc. We concentrate on the special case in which $v(d, x)=\widetilde{v}(d) x$.
Consider starting with a given $\widetilde{v}(d)$ and then reordering it to become $\widetilde{v}^{\prime}(d) .^{7}$ Let us say that a reordering $\widetilde{v}^{\prime}$ of $\widetilde{v}$ is weight increasing if the following condition holds. For any $d$ and $d^{\prime}$ such that $\widetilde{v}^{\prime}(d) \neq \widetilde{v}(d)$ and $\widetilde{v}^{\prime}\left(d^{\prime}\right) \neq \widetilde{v}\left(d^{\prime}\right)$, if $\widetilde{v}^{\prime}\left(d^{\prime}\right)>\widetilde{v}^{\prime}(d)$ then $P\left(d^{\prime}\right) d^{\prime} \geq P(d) d$.

The condition states that any values of $\widetilde{v}$ that have been reordered should be reordered so that higher values are assigned to degrees that have higher conditional weight.

PROPOSITION 6 (Weight Increasing Reorderings): If $\widetilde{v}^{\prime}$ is a weight increasing reordering of $\widetilde{v}$, then the corresponding $\phi^{\prime}$ and $\phi$ satisfy $\phi^{\prime}(x) \geq \phi(x)$ for all $x$, and so $\widetilde{v}^{\prime}$ generates greater diffusion than $\widetilde{v}$.

The implications of the Proposition are that in order to lower the set of tipping points and increase the set of stable equilibria, the appropriate choice of $\widetilde{v}(d)$ requires matching the ordering of $\widetilde{v}(d)$ with that of $P(d) d .^{8}$ The simple intuition is that in order to maximize diffusion, one wants the types that are most prone to adopt a behavior to be those who are most prevalent in the society in terms of being most likely to be neighbors.

Interestingly, this leads to conclusions that are counter to much of the common wisdom in the literature. Indeed, if one can target only a specific number of nodes, then one would like to target those with the highest degree, as they will have the greatest number of neighbors. This is the standard "hub" idea. Note, however, that the exercise here is different. We ask which types are most influential, when accounting for the population size and thus their likelihood to be neighbors.

Under a power distribution, $P(d) d$ is decreasing in $d$. So, in order to maximize adoption rates we would want a decreasing, rather than increasing, $\widetilde{v}(d)$. For a uniform degree distribution, the reverse holds. For a Poisson distribution, $P(d) d$ is increasing up to the mean and then decreasing

\footnotetext{
${ }^{7}$ Formally, $\widetilde{v}$ and $\widetilde{v}^{\prime}$ are reorderings of one another if there is a permutation $\pi$ of $1,2, \ldots$ such that $\tilde{v}[\pi(d)]=\widetilde{v}^{\prime}(d)$ for each $d$.

${ }^{8}$ Jackson and Yariv (2006) contains the proof, and a result for uniform $H^{c}$, where in order to increase $\phi$ pointwise, we do not need the reordering to be weight increasing, but rather just to increase weight on average.
} 
thereafter, and the ideal ordering of $\widetilde{v}(d)$ would match this shape, and be nonmonotonic.

\section{Convergence Patterns and $S$-Shaped Rates of Adoption}

We close with an analysis of convergence patterns. We can get an idea of the speed of convergence of the system at different points by examining the difference $x^{t+1}-x^{t}$, or $\phi(x)-x$, summarized as follows:

PROPOSITION 7: Let $H(d, x)$ be twice continuously differentiable and increasing in $x$ for all $d$. If $H(d, x)$ is strictly concave (convex) in $x$ for all $d$, then there exists $x^{*} \in[0,1]$ such that $\phi(x)-x$ is increasing (decreasing) up to $x^{*}$ and then decreasing (increasing) past $x^{*}$ (whenever $\phi(x) \notin\{0,1\}$ ).

\section{PROOF:}

Note that $[\phi(x)-x]^{\prime}=\sum_{d} \widetilde{P}(d)(\partial H(d, x) / \partial x)-$ 1 and $[\phi(x)-x]^{\prime \prime}=\sum_{d} \widetilde{P}(d)\left(\partial^{2} H(d, x) / \partial x^{2}\right)$. Let $x^{*}$ be such that $\left.(\partial H(d, x) / \partial x)\right|_{x^{*}}=1$, if it exists. When $H$ is strictly concave in $x$, if $\partial H(d, x) / \partial x$ $>1$ for all $x$ then set $x^{*}=1$, and if $\partial H(d, x) / \partial x$ $<1$ for all $x$ then set $x^{*}=0$. When $H$ is strictly convex in $x$, if $\partial H(d, x) / \partial x>1$ for all $x$ then set $x^{*}=0$, and if $\partial H(d, x) / \partial x<1$ for all $x$ then set $x^{*}=1$. Thus, if $H$ is strictly concave in $x$, then $[\phi(x)-x]^{\prime \prime}<0$. Therefore, $[\phi(x)-x]^{\prime}$ is positive up to $x^{*}$ and negative beyond it. The reverse holds for $H$ strictly convex and the result follows.

Proposition 7 helps characterize the diffusion paths. Consider a strictly concave $H$. There are three possible equilibria configurations: 0 is a unique and stable equilibrium; 0 is an unstable equilibrium and there is a unique stable equilibrium above 0 ; or 0 is not an equilibrium and there is a unique stable equilibrium above 0 . In the first case, $\phi^{\prime}(0) \leq 1$ necessarily and the dynamic process would converge to 0 regardless of the starting point. In the other cases, if $\phi^{\prime}(0)>1$, then $x^{*}$ lies above 0 and Proposition 7 implies that the adoption over time will exhibit an $S$-shape. From small initial levels of $x$ the change in $x$ will gain speed up to the level of $x^{*}$, and will then start to slow down

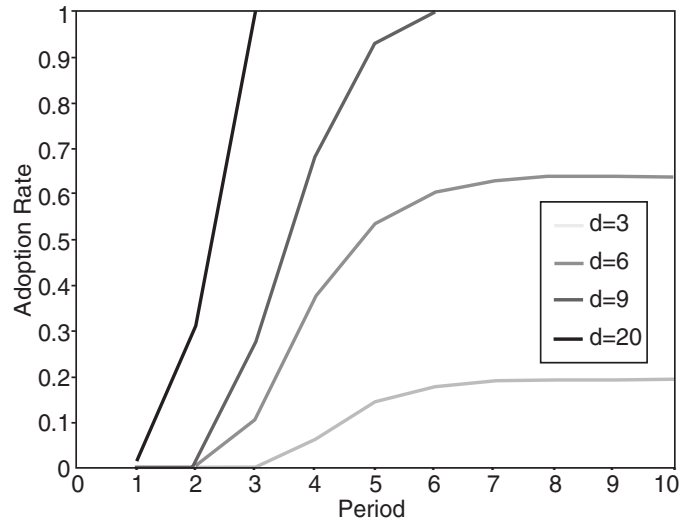

Figure 2. Fraction Adopting over Time by Degree

until eventually coming to rest at the adjacent stable equilibrium. ${ }^{9}$

We can also say something about adoption patterns by degrees. The dynamic process corresponding to each degree $d$ is given by $x_{d}^{t}=$ $H\left(d, x^{t-1}\right)$, where $x_{d}^{t}$ is the fraction of agents of degree $d$ who adopt at time $t$. In particular, whenever $v(d, x)=\widetilde{v}(d) x$, then $x_{d}^{t}$ exhibits the same curvature properties that are discussed above for $x$ itself. Moreover, the curves corresponding to the different $x_{d}^{t}$ are ordered according to $\widetilde{v}(d)$. In particular, for any stable point $x^{*}$, the corresponding distribution according to degrees is given by $x_{d}^{*}=\widetilde{v}(d) x^{*}$, and the curvature of $x_{d}^{*}$ follows that of $\widetilde{v}(d)$.

The distinction between different adoption paths corresponding to different degree players is important from an econometric point of view. Indeed, it provides additional restrictions on fundamentals arising from cross-sectioning data according to social degree.

As an illustration, consider the case in which $v(d, x)=d x$ and $H^{c}$ is uniform on $[c, C]$, so that $H(d, x)=\min [\max (0, d x-c), C-c] /(C-c)$. Figure 2 depicts the adoption dynamics corresponding to different degree agents in the case in which $c=1, C=5$, and the initial seed is 0.3 . In this example, higher degrees start adopting

\footnotetext{
${ }^{9} S$-shaped adoption curves are prevalent in case studies of diffusion. Frank M. Bass (1969) and follow-ups provided (network-free) contagion models explaining this general shape, and Peyton Young (2006) provides a learning model generating $S$-shaped adoption curves.
} 
the action 1 earlier and have steeper slopes early in the process; consistent with, e.g., the empirical observations on drug adoption by doctors in James Coleman, Elihu Katz, and Herbert Menzel (1966).

\section{REFERENCES}

Bass, Frank M. 1969. "A New Product Growth Model for Consumer Durables." Management Science, 15(5): 215-27.

Coleman, James S., Elihu Katz, and Herbert Menzel. 1966. Medical Innovation: A Diffusion Study. Indianapolis, IN: Bobbs-Merrill.

Galeotti, Andrea, Sanjeev Goyal, Matthew $O$. Jackson, Fernando Vega-Redondo, and Leeat Yariv. 2005. "Network Games." Unpublished.

Granovetter, Mark. 1978. "Threshold Models of Collective Behavior." American Journal of Sociology, 83(6): 1420-43.

Jackson, Matthew O., and Brian W. Rogers. 2007. "Relating Network Structure to Diffusion Properties through Stochastic Dominance." Advances in Theoretical Economics, 7(1): 1-13.

Jackson, Matthew O., and Leeat Yariv. 2005. "Diffusion on Social Networks." Économie Publique, 16(1): 69-82.
Jackson, Matthew O., and Leeat Yariv. 2006. "Diffusion of Behavior and Equilibrium Properties in Network Games." California Institute of Technology Division of the Humanities and Social Sciences Working Paper 1264.

López-Pintado, Dunia. 2004. "Contagion in Complex Networks." Unpublished.

Morris, Stephen. 2000. "Contagion." Review of Economic Studies, 67(1): 57-78.

Morris, Stephen, and Hyun Song Shin. 2003. "Global Games: Theory and Applications." In Advances in Economics and Econometrics: Theory and Applications, Eighth World Congress, Vol. 1, ed. Mathias Dewatripont, Lars P. Hansen, and Stephen J. Turnovsky. Cambridge: Cambridge University Press.

Newman, Mark E. J. 2002. "The Spread of Epidemic Disease on Networks." Physical Review E, 66(1): 016128 [11 pages].

Pastor-Satorras, Romualdo, and Alessandro Vespignani. 2000. "Epidemic Spreading in ScaleFree Networks." Physical Review Letters, 86(14): 3200-203.

Rogers, Everett M. 1995. Diffusion of Innovations. New York: Free Press.

Young, H. Peyton. 2006. "The Spread of Innovations by Social Learning." Unpublished. 